\title{
SOME RESULTS OF THE SPACE DISTRIBUTION OF THE ACTIVATION VECTOR OF THE FILAMENTS IN THE \\ 11-YEAR CYCLE
}

\author{
V. V. KASINSKIJ, G. Ya. SMOLKOV, and G. N. ZUBKOVA \\ Sibizmiran, Irkutsk, U.S.S.R.
}

In order to obtain new information on the manifestation of basic mechanisms of solar activity with the sunspot cycle development we have studied peculiarities of the latitude-time distribution of activation positions and suddenly disappearing filaments with respect to sunspot groups of cycles 18 to 20 .

Interest in such an analysis is caused by the particular role of filaments which is conditioned by their close relationship with the appearance and variation of sunspot groups, by the development of flares, topology and magnetic field disturbances, as well as by the development of coronal transients in the solar atmosphere, detected by 'Skylab'.

Assuming that activation and a sudden disappearance of filaments (later on, simply activation) are triggered by the sunspots, or flares influencing the former along the line between them or along the filament, we have chosen, in terms of coupling parameters, differences in their latitudes $(\Delta \varphi)$ and longitudes $(\Delta \lambda)$, or displacement vectors $(\bar{R})$ produced in them, after which $\Delta \varphi-, \Delta \lambda-$, mean-diagrams were then constructed. The activated filaments were identified with the neighbouring sunspot groups up to a distance $R$ less than $25^{\circ}$ in latitude and longitude. Those active regions were chosen as the triggering centres for which $R$ has a local minimum. An analysis of these diagrams yielded the following results.

\section{Averaged $\varphi t$-Diagrams of Activation}

The regularity of the filament activation position distribution with respect to sunspot groups with the phase of the sunspot cycle evolution, is indicated by the unaccidental character of this phenomenon:

(a) The distribution looks like a butterfly diagram which presents the mean value filament activation position. This distribution is closely associated with other phenomena of solar activity and reflects the effect of basic laws and mechanisms of the solar activity development (Figures 1 to 3 ). On all pictures the scale of vectors corresponds to the scale of latitude.

(b) There are two latitude zones of filament activation phenomena with the cycle evolution, marked in both their filament displacement direction and magnitude with respect to sunspot groups: high-latitude and near-equator zones have filament activation displacement (represented by small arrows) toward the poles and toward the equator, respectively. In the high-latitude zone displacements are greater than in the near-equator zone. 
ب० 1945 1955

1960

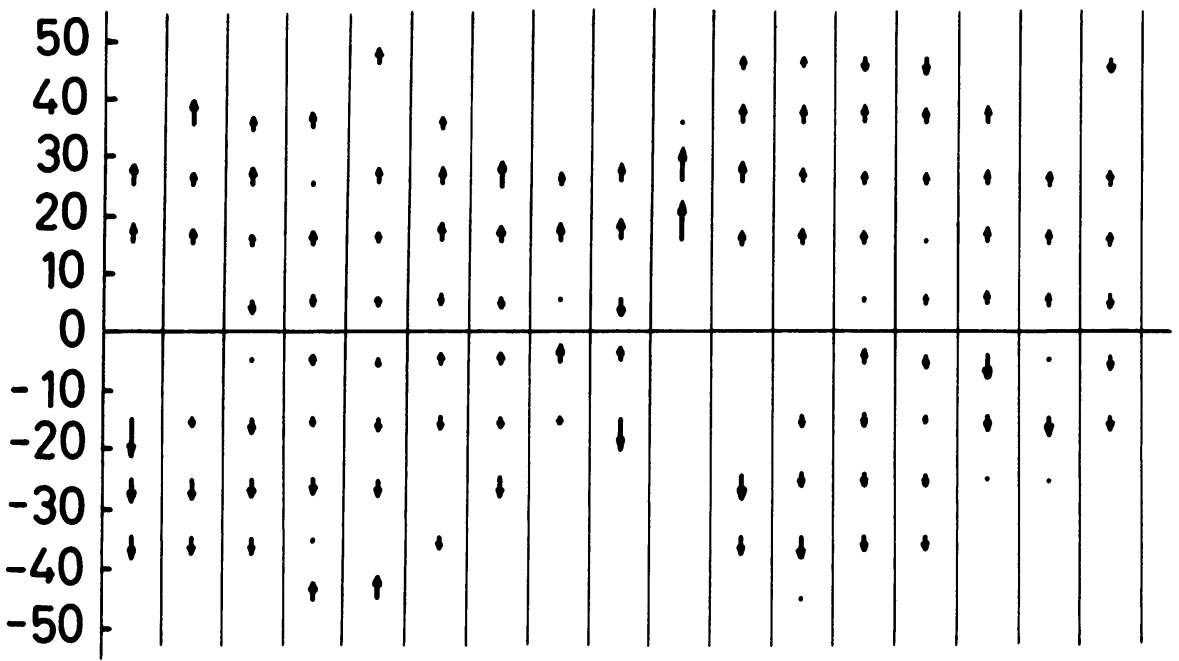

Fig. 1. The diagram of the latitudinal (meridional) displacements of the sudden disappearances of filaments, 1945-1962, cycles Nos. 18, 19.

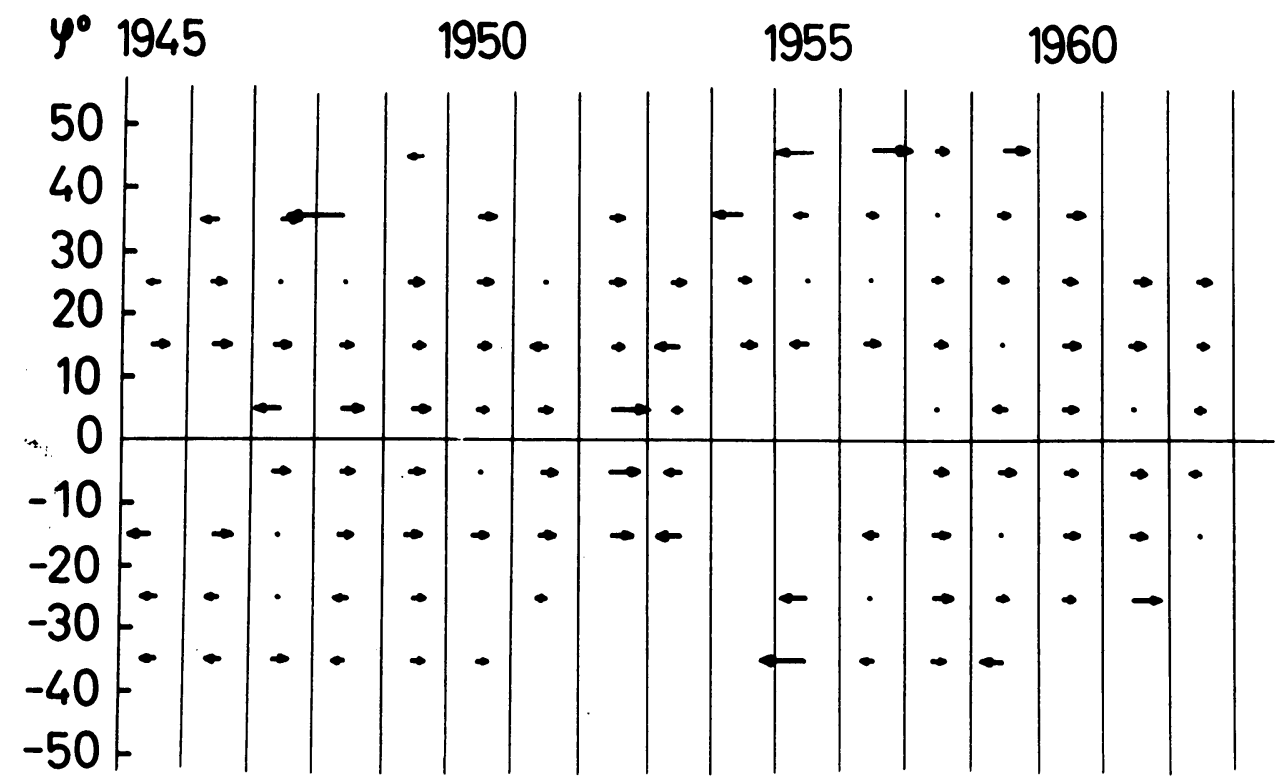

Fig. 2. The butterfly diagram of the longitudinal displacements of the sudden disappearances of filaments, 1945-1962. 


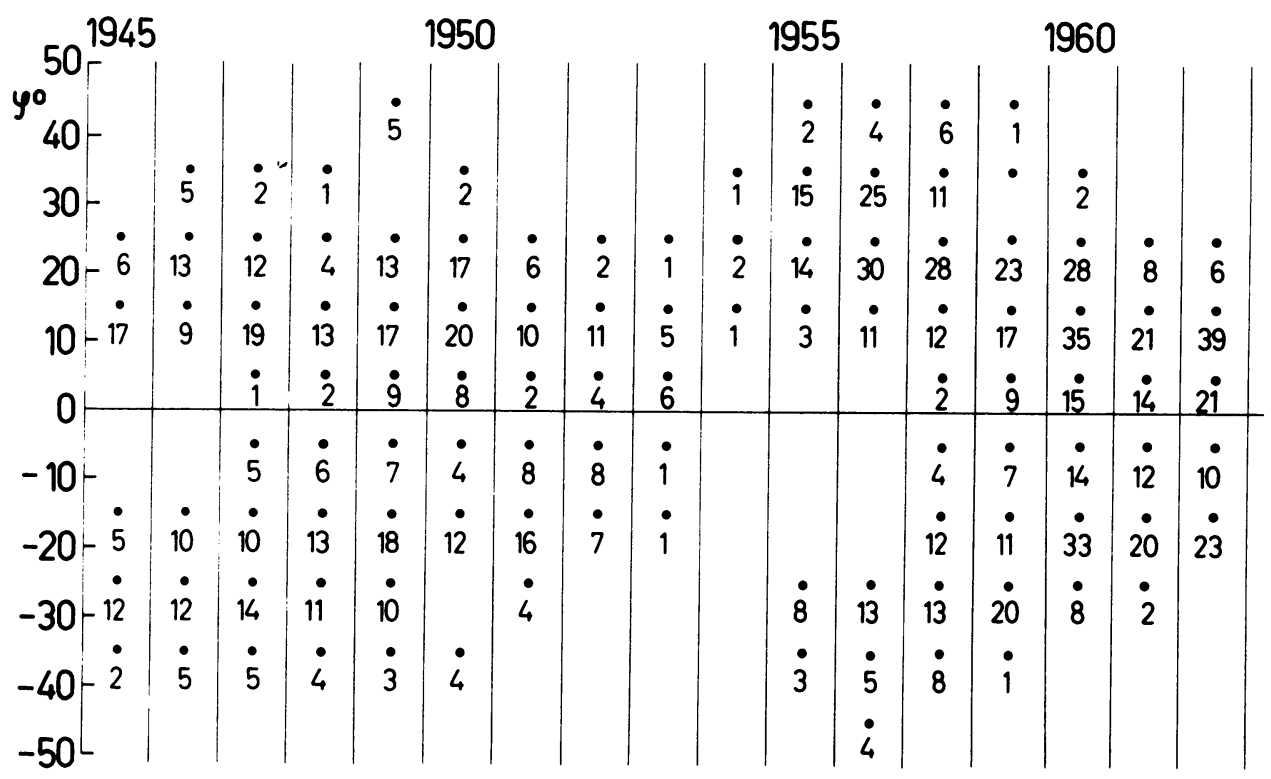

Fig. 3. The butterfly diagram of the total number of activations.

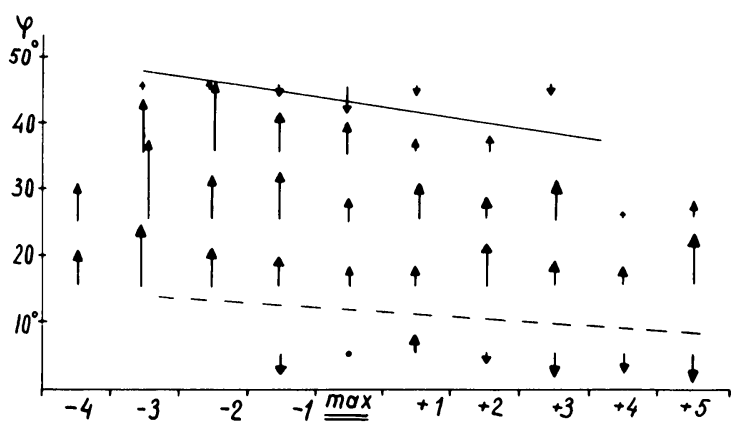

Fig. 4. The mean diagram of the latitudinal displacements of the filament activations for the single solar hemisphere.

Polar filament activation displacements are the largest at the increasing phase of the solar cycle, then they decrease. The near-equator type of displacements is more pronounced at the decreasing activity phase (Figure 4).

In addition, during the whole cycle, two marked zones of sign change of latitude displacement are retraced. The high-latitude, or conversion zone is at the latitudes near $45^{\circ}$, and the low-latitude or divergation zone is in the latitude range of $8-10^{\circ}$. Outside these zones, the equatorial type of displacement is observed, inside the zone, polar displacements are seen. 


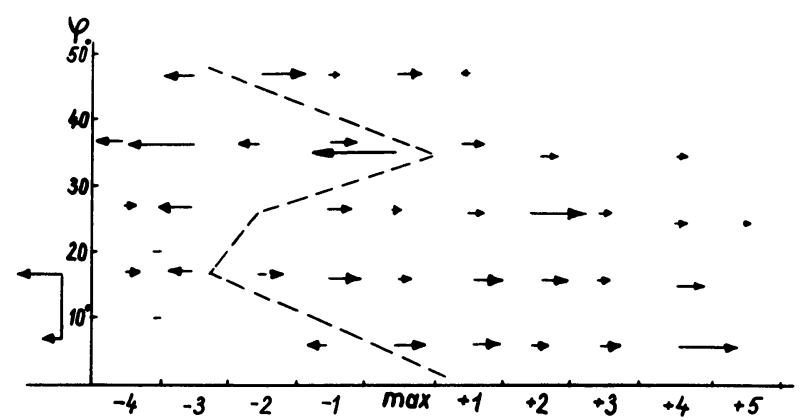

Fig. 5. The average diagram of the longitudinal displacements of the filament activations in one hemisphere.

(c) The directions of filament activation displacement in longitude vary with the cycle evolution from an eastward direction at the beginning of the cycle to a westward one at the end. In the cycle maximum such displacements are less noticeable than during minima epochs of solar activity (Figure 2).

The transition from the eastward displacement to the westward one takes place not gradually, but as it is seen from the behavior of the zero line at Figure 5, by fits and starts and resemble displacement or torsional oscillations. Variations in sign change of displacement are found only on the ascending branch of the sunspot cycle when interaction between the fields of the preceding and the present sunspot cycles still takes place. After the maximum, only westward displacements are observed.

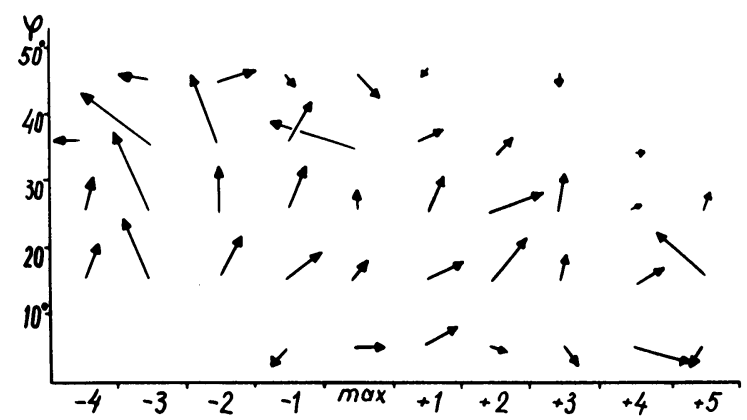

Fig. 6. The constructed latitudinal-longitudinal diagram of activation displacements for one hemisphere.

(d) The directions of displacement vectors tend, with the cycle evolution, to vary gradually from the polar-eastward to the polar-westward sense in the high-latitude zone, and from equatorial-eastward to equatorial-westward sense in the low-latitude zone (Figure 6). The polar type of displacements is predominant. Therefore, displacement vector staggering is observed instead of simple displacement direction change, found by one of the authors for the flares $[1,2]$. 


\section{The Fine Structure of $\varphi$ t-diagrams of Activation}

The fine structure of $\varphi t$-diagrams (Figure $7 \mathrm{~b}$ ) together with the mentioned (average) common features may be shown by means of sum $(N+S)$ and subtract $(N-S)$ two pictures in the $N$ and $S$-hemispheres thus drawing the zero line separating the region of polar displacements from the equatorial ones. Both zones exist simultaneously through the whole cycle. The equatorial zone, extending up to latitudes of $20-30^{\circ}$ at the beginning of a cycle, reveals itself more completely after the activity maximum. During the 20th cycle the zero line has a deviation to the high latitudes by 1966-68. This fact is one more piece of evidence for an anomaly in the 20th cycle, noted earlier from other phenomena of solar activity (sunspots, polar prominences, corona).

The filament activation position distribution in the northern and southern hemispheres is marked by considerable asymmetry (Figure 7a). This asymmetry is stronger at the beginning of a cycle, and at high latitudes during practically the whole cycle. At intermediate and low latitudes, especially after the maximum epoch, nearly symmetric distribution of activation takes place (vectors tend to zero).

The distributions of activation displacements modulus with respect to latitude are appreciably different from year to year (Figure 8), also exhibiting asymmetry of hemispheres. Most activations take place in the northern hemisphere. In it, at the

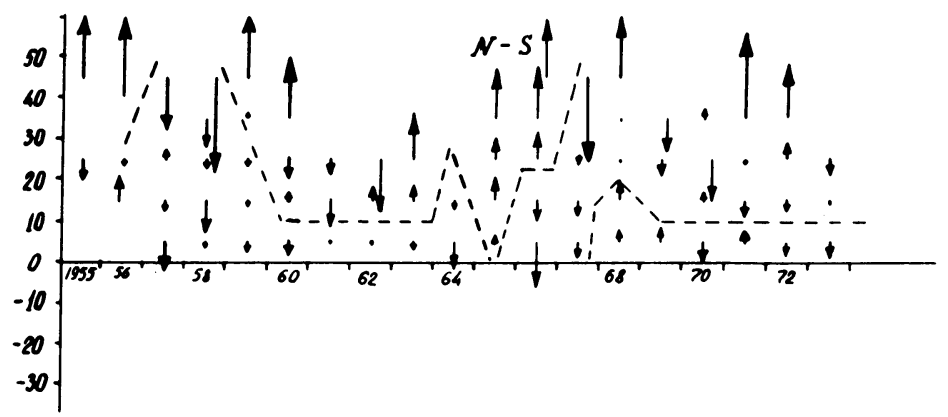

(a)

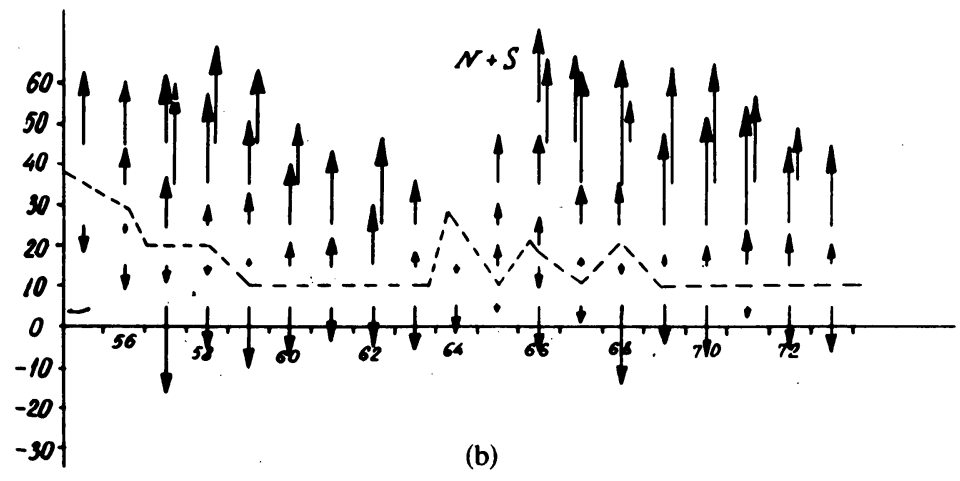

Fig. 7. The fine structure and the zero line asymmetry drift for the meridional displacements of the filament activations in the 19th and 20th cycles, 1955-1973. 


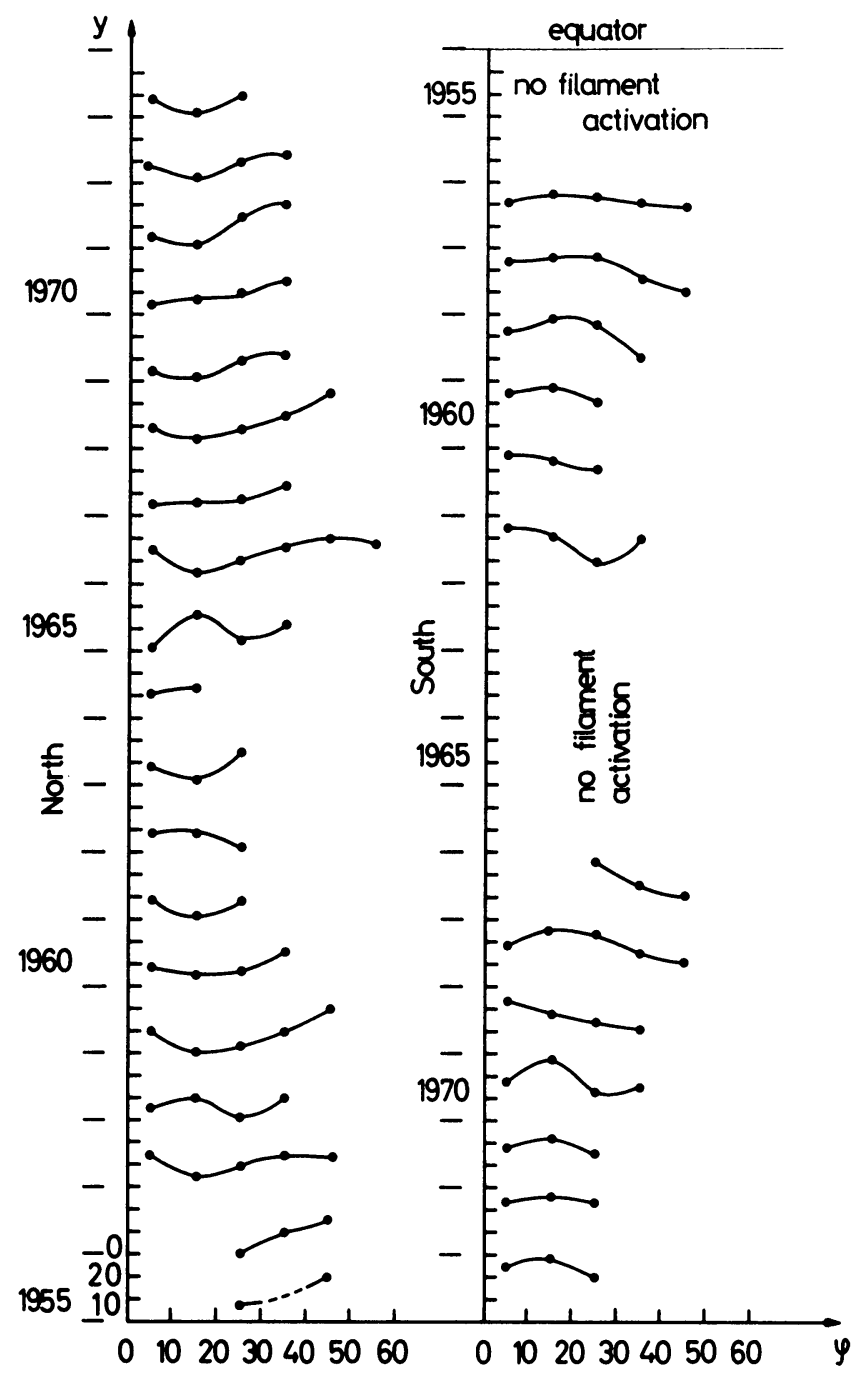

Fig. 8. The changes of modulus of distance 'sunspot group - activation of filament' within the time of the cycle in both hemispheres of the Sun, 1955-1973.

latitude of $15-20^{\circ}$, a marked minimum of activation displacements is observed. Later on, displacements increase with latitude. In the southern hemisphere (less active) such a minimum is less pronounced. During some years, it is observed at higher latitudes $\left(25-30^{\circ}\right)$ than that in the northern hemisphere, an effect which seems likely to be associated with cycle delay in the southern hemisphere.

When summarizing we have obtained the following results.

(1) On the material of Cartes Synoptiques de la Chromosphère Solaire the proper position of the active filaments relative to the adjacent sunspot's group (D.B. phenomena) are considered [3,4]. The vector-displacement for each activation of 
the filament (each D.B.) are summarised inside the space-time region on the time-latitude diagram along the latitudinal intervals $\Delta \varphi=10^{\circ}$ and time $\Delta t=1 \mathrm{yr}$ of the cycle.

(2) The 'butterfly diagram' constructed has been analysed from different points of view. The two populations of active filaments are picked up. One of them is the equatorial zone filaments and the other is the middle and high latitude ones. The middle-high latitude type shows regular rotation of the proper vector from the polar-eastern direction to the polar-western in the end of cycle. In the middle of the 11 -year cycle there is a well-defined polar direction of the vectors. For the different latitudinal zones the vector $\bar{R}$ is constructed (1945-1973).

The results obtained are discussed from the point of view of common regularities of 11-year activity of the sunspots and the filaments on the Sun.

The full text of this report will be published in Issled. Geomag. Aeron. Fiz. Solnza 38 (1976), 225.

\section{References}

[1] Kasinskij, V. V.: 1973, Solar Data Bulletin, NN 2, 7, 1973.

[2] Kasinskij, V. V. and Plusnina, L. A.: 1974, Issled. Geomag. Aeron. Fiz. Solnza 31, 44.

[3] Cartes Synoptiques de la Chromosphère Solaire, Méudon, 1945-1973.

[4] Quart. Bull. Solar Activity, Zürich, 1955-1973. 